Research Article

\title{
Internet Rumor Reporting System Based on the Blockchain Incentive Mechanism
}

\author{
Jie Bai $\mathbb{D}^{1,2}$ Yanhui Du $(\mathbb{D})^{1}$ and Tianliang $L u \mathbb{D}^{1}$ \\ ${ }^{1}$ School of Information Technology \& Network Security, People's Public Security University of China, Beijing 100038, China \\ ${ }^{2}$ College of International Education, Wenzhou University, Wenzhou 325035, China \\ Correspondence should be addressed to Jie Bai; 20170166@wzu.edu.cn
}

Received 24 August 2021; Revised 19 September 2021; Accepted 25 September 2021; Published 18 October 2021

Academic Editor: Sang-Bing Tsai

Copyright (c) 2021 Jie Bai et al. This is an open access article distributed under the Creative Commons Attribution License, which permits unrestricted use, distribution, and reproduction in any medium, provided the original work is properly cited.

\begin{abstract}
This study examined the characteristics of internet rumors in the user-generated content (UGC) mode, analysed the advantages of using the blockchain technology to curb internet rumors, and proposed a framework of the internet rumor reporting system based on the blockchain incentive mechanism. With Truffle as a development framework, intelligent contracts such as create, read, update, and delete were created using Solidity. The end users were connected to the blockchain using the MetaMask plugin. Finally, rewards were obtained by the participants, and the decentralizing program Dapp was created. The results showed that the TRUES could support multiuser reporting and execute intelligent contracts automatically and efficiently in a complex internet rumor environment; for the range of 1-2896 participants, virtual currency rewards could be obtained in $0.5-2.5 \mathrm{~d}$. The conclusion showed that the internet rumor reporting system under the blockchain incentive mechanism had the characteristics of multilevel management, and distributed ledger and digital signatures contributed to the retention and traceability of rumors. Intelligent contracts and consensus mechanisms have obvious advantages in dealing with the complex forms of internet rumors.
\end{abstract}

\section{Introduction}

It is reported that the number of internet users has reached 989 million in China, with 927 million video users accounting for $93.7 \%$, according to the 47 th "Statistical Report on China's Internet Development Status" released by China Internet Information Center in February 2021 [1]. The proportion of user-generated content (UGC) mode network information dissemination is gradually increasing with the rapid development of communication technology. The generation, diffusion, and even public opinion fermentation of network rumors can be easily caused by UGC information because of their characteristics of subject decentralisation, content fragmentation, and fission propagation speed in the propagation process. Network rumors are generally spread through microvideos, fake screenshots, forged pictures, video reprocessing, and other forms to which public are attracted. The reason of creators to spread network rumors is complex. The lack of judgement makes more audience as secondary rumor spreaders. Rumors that spread in the form of "video + pictures" are more attractive to the audience and more difficult to control.

Network rumors refer to the false information that are transmitted through the network media (for example, microblog, website, forum, and social software) and reflected in multimedia forms such as text, pictures, and voice-video. Text information in network rumors can be divided into thematic text and emotional tendency text [2]. Texts with evident words such as violence, pornography, fraud, and garbage information are judged as thematic texts. Web crawler technology is commonly used to capture specific keywords and shield the captured messages. The rumor with emotional tendency contains the information of the publisher's views, attitudes, and positions. Therefore, the information is concealed and is difficult to be graphed by the crawler program. Artificially identifying rumors is manually marked by the network security department, which consumes extensive manpower and is difficult to meet the requirements of control. Therefore, more accurate traceability and intelligent mining methods must be developed. 
Artificial intelligence provides multiple tools for the excavation of general network rumor information (including text, image, and voice). For example, deep learning is widely used in text classification in natural language processing $[3,4]$, and neural networks are used for the identification of illegal images [5]. However, with the advent of the UGC era, rumors based on video and live broadcast mode have large scene changes; therefore, these are difficult to recognise. A real-time frame-cutting transmission recognition is required with a large number of manual judgements; therefore, it is difficult to obtain and analyse public opinion data.

Blockchain is applied to the field of public opinion control and electronic certificates, with advantages of distributed database technology, decentralisation, and consensus mechanism $[6,7]$. However, the earlier studies mainly use the existing blockchain websites to process the generated text and picture data at a later stage, which lacks immediate regulation $[8,9]$. Therefore, we must immediately exploit the advantages of the blockchain technology, improve the original rumor processing mode, and establish an efficient real-time rumor reporting system to warn and deal with rumors that endanger social security.

The main innovations of this study are as follows:

(1) This study proposes a network rumor discovery model, TRUES, under the blockchain incentive mechanism. Each participating node is encouraged to submit the rumor information of its initial judgement. Certification is implemented through smart contracts when the network rumor information reaches a consensus among the participating nodes in the blockchain. Public surveillance is encouraged, and the sources of rumor data are broadened by this mechanism.

(2) In the process of blockchain Dapp creation, the traditional method is improved to avoid separating the storage layer code from the application layer code. Intelligent contracts simplify independent database components and use codes to complete reading and data storage functions. This more flexible setting overcomes the problem of occupying storage space owing to heavy workload.

\section{Advantages of the Blockchain Technology in Network Rumor Processing}

As a distributed accounting technology, the advantages of the blockchain decentralisation and consensus mechanism provide a variety of possibilities for its application in the public opinion prevention and control model. The decentralised model proposes a new method to judge and prevent rumor spreading. The original rumor processing path is generally from the initial publisher $\longrightarrow$ information terminal $\longrightarrow$ network control centre $\longrightarrow$ public network security department $\longrightarrow$ network control centre $\longrightarrow$ information terminal $\longrightarrow$ feedback for the published content [10]. The decentralised model can overcome the disadvantages of the original centralised control of public opinion, such as multiple links, considerable time, heavy tasks, and a large deployment of police.

Another advantage of the blockchain technology is the consensus mechanism. Entrants can be used as nodes on the blockchain; each node has the function of identifying rumors and backing up information. When a participant receives specific information, it uses common sense to judge the authenticity of the information, broadcast it if it is true, and reach a consensus after mutual verification with other nodes; then, the information is added to the chain. Information is filtered and blocked based on the consensus mechanism when more than $50 \%$ of the nodes in the entire chain judge it as a rumor. To achieve the purpose of instant processing, an integrated data link is formed, which provides technical support for later traceability.

The intelligent contract can meet the high efficiency requirement of rumor processing. The intelligent contract contains all the information about the transaction and automatically executes the resulting operation after meeting the requirements; hence, trust can be built on the basis of the algorithm, which is much higher than that of the human value judgement. Furthermore, the spontaneous execution of the intelligent contract based on automatic hosting considerably reduces the labour cost. For example, the preliminary research by Sheng et al. [8] and Pandou and Chunhua [9] mainly used the existing blockchain websites to process and test the generated text, picture, and network rumor data but lacked immediate regulatory countermeasures. Xiwei et al. [11] tested the design of the intelligent contract on the well-known blockchain Steemit platform by adding the common rule formulation of users on each node. The support and opposition of platform nodes to the information released by other nodes will affect the ranking of their information and the communication efficiency, which provides a basis for the platform to deal with false information or low-value information. Sulin et al. [12] constructed and conducted simulation experiments on the three-stage network rumor screening model of public opinion outbreak, public opinion fermentation, and public opinion diffusion and proved that the model can ensure the traceability of public opinion information, but it is only verified for a single platform and ignores the common fermentation characteristics of rumor information on multiple platforms. Weichong et al. [13] analysed the information acceptance behaviour of users in public opinion in combination with the blockchain technology and built and tested the public opinion platform, but it also lacked cross-platform function. Dan et al. [14] analysed the blockchain technology from the perspective of multisource information, but the information of this test comes from the scientific and technological literature platform, and its variability and timeliness are far lower than those of rumor information. It is worth mentioning that Zhao [15] and others analysed the law of network public opinion information dissemination under the blockchain environment, which laid a foundation for the research of the incentive mechanism on user behaviours.

The digital signature technology of the blockchain reduces the possibility of information tampering. If a rumor 
publisher wants to disseminate by forging real information, he must obtain the private key from the original information publisher, and the corresponding hash value will lead to the change of the digital signature. Blockchain automatically verifies the changed digital signature through intelligent contracts; therefore, the generation and traceability of rumors will be reported in time.

In the blockchain 3.0 industry application era, timestamp technology can fully guarantee the entire process of information dissemination in rumor prevention and control [16]. The intelligent contract and consensus mechanism improve the accuracy and efficiency of data processing in rumor dissemination. The unique technical characteristics of the blockchain can fully improve the accuracy and efficiency of network rumors' management and can provide effective tools for the discovery and blocking of network rumors in the UGC era.

\section{Network Rumor Reporting Model in the Blockchain Incentive Mechanism}

The core of the blockchain is the incentive mechanism. In the bitcoin system, the essence of the reward is that miners (nodes) obtain established interests by participating in transactions. One study has theoretically confirmed that economic benefits affect user forwarding [14]. Each participating node in the system submits its identified rumor information. The submitted network rumor information reaches consensus in the participating node of the blockchain through an intelligent contract; it is performed through an intelligent contract such that the participating node will obtain a reward for virtual currency (financial assets) [17]. This incentive mechanism enables users to more actively report and share rumor information, which can considerably transform rumor entrants and observers in the process of information dissemination into rumor whistleblowers. In this section, we introduce the construction and application test of the network rumor reporting model in the blockchain incentive mode.

3.1. Network Rumor Mining System Model under the Blockchain Incentive Mechanism. The "TRUES" system is different from the traditional online rumor reporting system. All business logics of the traditional rumor reporting system are defined and implemented on specific private servers (internet companies or public security departments). The business of "TRUES" is defined and implemented in the intelligent contract on the decentralised Ethernet blockchain, which is more secure to avoid the impact of server problems on the "TRUES" system.

The assumptions of "TRUES" are as follows:

(1) Initially, an ETH is assigned to each participant, which can be paid to the contract for joining the "TRUES" competition

(2) "TRUES" begins when the first participant enters

(3) Contract is pinged by entrants every day
(4) If a participant does not ping contract in $24 \mathrm{~h}$, other users can delete it

(5) The last participant will obtain all virtual currencies from the contract when the whole process is completed

The structure of the "TRUES" system is demonstrated in Figure 1.

3.1.1. Introduction of Truffle for the TRUES System. This study used the current mainstream development framework, Truffle, with the advantages of simply compiling, testing, and deploying contracts to the blockchain. The front end can be quickly set and connected to a deployed contract. It can perform automated contract tests and deploy network management to any number of public and private networks in application, with interactive consoles for direct communication. In this study, npm install-g truffle@4.0.4 version was installed.

3.1.2. Intelligent Contract. Solidity was used to create intelligent contracts such as create, read, update, and delete to better manage the accounts of entrants in the system. This contract is the basic operation that we require to perform on the storage of intelligent contracts when managing entrants.

An intelligent contract provides the ability to initialize a permanent state and read and store data through its code. The contract uses state variables instead of the default global variable in permanent storage. The default location depends on the type of variable it involves.

Database: using the mapping structure, indexes all registered entrants according to their addresses.

A new type of Centrants, containing various contract operations, is set.

Create: create the contract after completing the storage initialization; create implies adding new items to the permanent storage structure (participant mapping). This function allows new adders to register themselves in the database.

Read: define the findentrants method in the centrants contract and read the mapping record according to the specified primary key (participant address).

This function will provide entrants' addresses instead of direct access to player details from the mapping loop. Owing to the setting of network security, entrants authenticate their identity through their phone number.

Update: update or edit the existing entries in the participant mapping storage. Each participant maps its detailed information to its addresses such that we only need to target ID in the mapping and update the attributes of its corresponding structure. The old value will not be deleted or rewritten when an item is updated. A new value contained in the current block is defined, while the old value is put into the previous block. This reflected the untamperability feature in TRUES. 


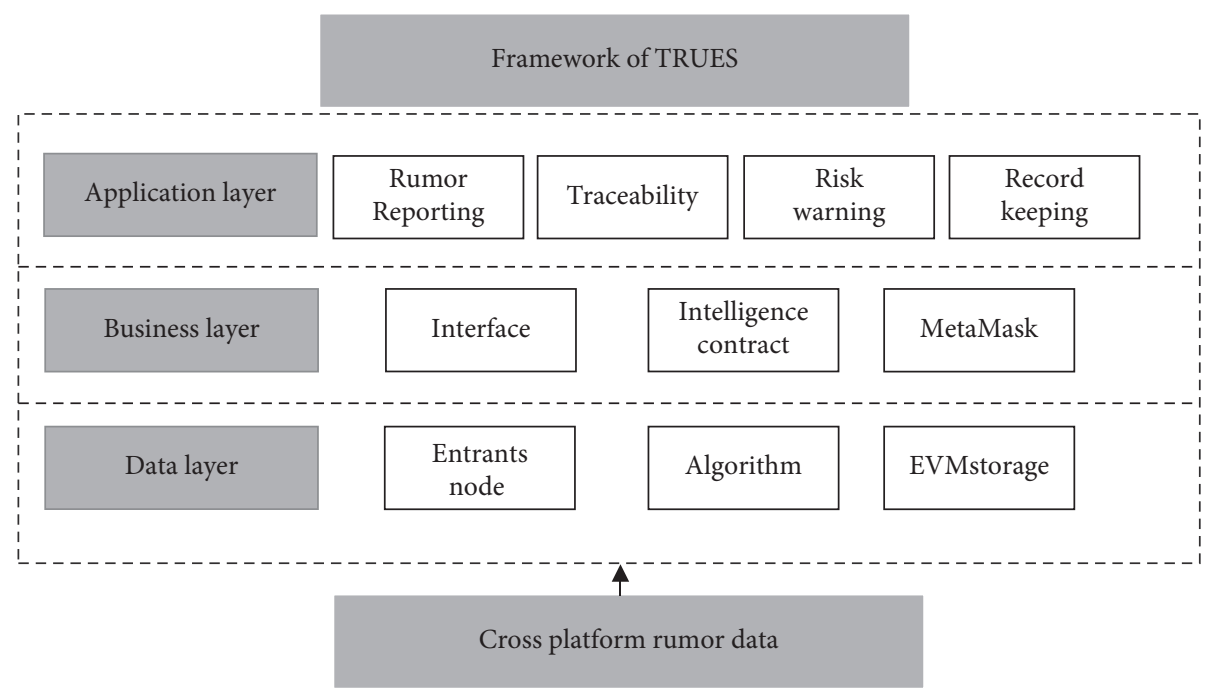

FIgURE 1: Structure of the TRUES system.

Delete: used to stop or delete existing entries.

Removeentrants: deletes the elements at a specific index (address). In the mapping, the specified key (address) is set to zero.

3.1.3. Establishment of a CTRUES Contract. Implement the interface with the keyword.

Join function allows entrants to join the ongoing TRUES system. If entrants are declared as payable, they can receive Ethernet currency.

Ping function: maintains the track record of participant activity ping

Eliminate function: removes inactive entrants

ClaimReward: sends the total awards under contract control to the last active participant

New ActiveentrantsEv and eliminateentrantsEv are two events when new entrants join the system or when entrants are eliminated from the system, respectively.

Lindex tracks the index of the previous active user as an integer variable.

3.2. Adding, Eliminating, and Obtaining Rewards in the Operation of the TRUES System. At the beginning of the incentive process, the entrants invoke the join method and send an Ethernet currency to the True contract.

Entrants must meet the following conditions for joining TRUES.

Entrants should be registered in the contract and send sufficient money. msg value $\geq 1$ ether.

Entrants did not join the ongoing TRUES system. msg value $\geq 1$ ether and Tpension [msg.sender] $=0$.

The Ether coins we provide are stored in Tpension.

3.2.1. Rumor Reporting Function. After performing the join (.) function, use ping (.) to allow entrants to update their activities. Entrants send a transaction call ping to prove their activity.

3.2.2. Function of Eliminating Inactive Entrants and Sharing Rewards. Entrants can trigger the eliminate function to ensure that the system can eliminate participants who have no ping contract in the past $24 \mathrm{~h}$. This function will delete inactive participants from the list of active participants and set the participant ID to zero. Furthermore, Pension function is to share the funds of the eliminated participants.

3.2.3. Obtain Reward Function. In the final stage of rumor reporting, the winning entrants obtain rewards by calling the ClaimReward function, which is realized by the function of distributing funds every time after the previous smart contract eliminates inactive participants.

\section{Application Test of the TRUES System}

MetaMask connects the end users to Ganache (blockchain) such that users can use and manage their funds.

In the Drizzle box folder, run the command npm run start.

The user interaction interface of the TRUES is shown in Figure 2.

To test 22 entrants in an ongoing online rumor, entrants must register using real information such as a name or phone number. Click Submit. In MetaMask, open the first pop-up window. Consider the 22nd account as an example. The initial account setting of the TRUES system is shown in Figure 3.

After 22 entrants in the test group join the TRUES system, click commit for each account successively. Renew the intelligent contract by reporting rumors.

When the join and renewal operations are completed, Drizzle will automatically update the ContractData component instead of refreshing the entire page. In the test process, time can be increased to eliminate some entrants. To 


\section{Authenticate Account}

Detailed address:

72-34-15-76-25

Entrant Contract

Register

Authentic Name

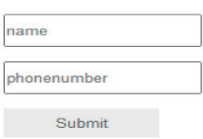

Ctrues

Last Ping: 12581111119

bonus: 20000000000000000

1.111 Ether

join TRUES

(only the fitst time)

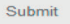

Ping :

(ping interval is unit time)

Submit

Eliminate an opponent

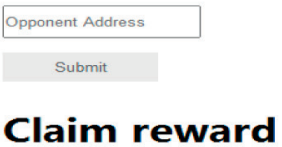

Submit

Figure 2: TRUES system user interface.

enable the Truffle console in a new terminal, generate an interactive console connected to Ganache, and run RPC calls. After $24 \mathrm{~h}, 21$ entrants are eliminated, and Drizzle automatically displays details at the bottom of the page when entrants are eliminated. The phase-out account information is shown in Figure 4; the remaining entrants will receive their due reward in Ethercoin.

By increasing the number of tests, the number of entrants is increased to 267 with the rumor reporting time set for $2 \mathrm{~d}$. Finally, 266 entrants are eliminated. The ETH reward obtained by the winner is shown in Figure 5.

As the blockchain technology is in the development stage without national test standard at present, this study adopted the current popular standards of China blockchain technology and industrial development forum to conduct a comprehensive system test for the program, including the function test and performance test with several participants. With the increase in the number of participants and test time, the functions of the TRUES system could meet the business needs and accurate access. The consensus mechanism and intelligent contract could support tamper-proof and content upgrade. The rumors reported by participants came from 2-20 platforms. The verification results showed that the TRUES system had good stability and adaptability in dealing with network rumors in the cross-platform mode.

According to the performance test results of the TRUES system, multiple account tests can run stably. To test the stability of the system with the number of participants gradually increasing, the stage test results are shown in Table 1. According to the network rumor spreading practice, the duration range from attracting audience interest to gradually withdrawing from hot search is $0-3 \mathrm{~d}$; the test time was set within $3 \mathrm{~d}$. The test results showed that the TRUES system could adapt to continuous reporting and could distribute rewards in time.

In summary, the advantage of TRUES is to reduce memory consumption by mapping storage. Intelligent 


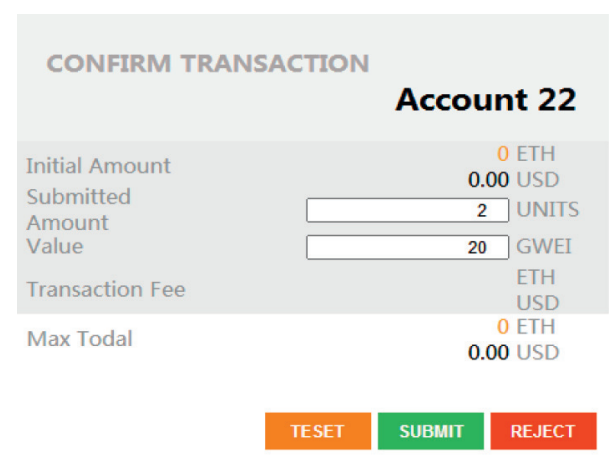

FIGURE 3: Initial account setting of the TRUES system.

Eliminate an opponent
account:
NO.i
phone number:
1567890123
detailed address:
0x-7D-75-97-22

Figure 4: Phase-out account information in TRUES.

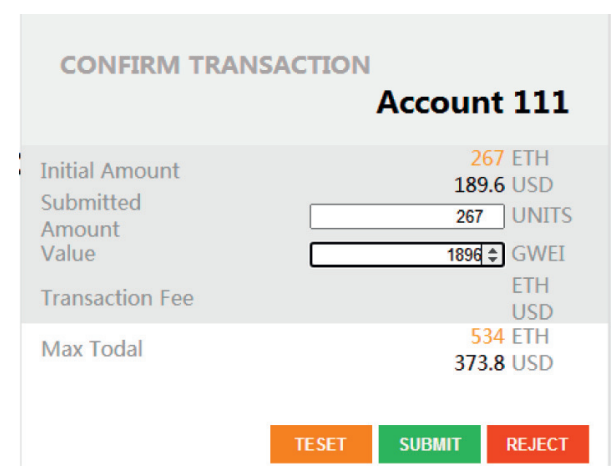

FIGURE 5: Reward interface of the TRUES.

TABle 1: Phase test results.

\begin{tabular}{lcc}
\hline Number of participants & Time $(\mathrm{d})$ & Result \\
\hline 22 & 0.5 & Stable \\
222 & 1 & Stable \\
997 & 1 & Stable \\
1997 & 2 & Stable \\
2896 & 2.5 & Stable \\
\hline
\end{tabular}

contracts could eliminate negative participants and provide rich rewards to active participants quickly and accurately. The verification results showed that the TRUES had obvious advantages in dealing with network rumors under the UGC mode. The harmfulness of rumor multiplatform is ignored by the traditional way for internet rumor handling. The TRUES system based on the incentive mechanism is suitable for providing rumor clues from different media platforms, which can better meet the comprehensive judgement of the multiplatform.

\section{Conclusions}

This study analysed the characteristics of network rumors, including information subject decentralisation, content fragmentation, and fission communication under the UGC mode. Combined with the advantages of the blockchain incentive mechanism, the TRUES rumor reporting system was optimized by multiuser testing. It was proved that users in the TRUES system could obtain virtual currencies by reporting rumors, which could mobilize the enthusiasm of rumor whistleblower and broaden the source of rumor data. The blockchain technology can provide identification, processing, and warning in the entire process to meet the needs of online rumor reporting efficiently and precisely. Therefore, TRUES has wide social needs, high technical feasibility, and good application prospects.

\section{Data Availability}

The data used to support the findings of this study are available from the first author upon request.

\section{Conflicts of Interest}

The authors declare that they have no conflicts of interest.

\section{Acknowledgments}

This study was supported by the National Key R\&D Program Project (2016YFB0801105) and Construction Project of High-Level Nonediting Scientific Research Institutions of People's Public Security University of China (2021fzb13).

\section{References}

[1] China Internet Network Information Center (CNNIC), Statistical Report on China's Internet Development, Internet Information Center, Beijing, China, 2021.

[2] Z. Haiquan, L. I. Ronglu, and H. U. Yunfa, "Polarity text filtering based on semantic analysis," Journal of Communication, vol. 25, pp. 78-85, 2004.

[3] Z. Qingqing, Research on Text Sentiment Classification Based on Machine Learning, Northwestern Polytechnical University, Xi'an, China, 2016.

[4] Y. Yan, Text Representation and Classification with Deep Learning, University of Science \& Technology Beijing, Beijing, China, 2016.

[5] F. Yi, Pornographic Image Recognition Method Integrating Local Sensitive Feature Detection and Global Perceptual Classification, Beijing Jiaotong University, Beijing, China, 2020.

[6] Z. Weijie, "Blockchain technology: development and prospects," National Science Review, vol. 6, pp. 193-197, 2019.

[7] Z. Zheng, S. Xie, H.-N Dai, X. Chen, and H. Wang, "Blockchain challenges and opportunities: a survey," International Journal of Web and Grid Services, vol. 14, pp. 352375, 2018.

[8] B. I. N. Sheng, S. U. N. Gengxin, and Z. Shuang, "Public opinion propagation model in social network based on blockchain," Journal of Applied Science, vol. 37, pp. 91-202, 2019. 
[9] J. Pandou and Y. Chunhua, "Research on characteristics and rules of information transmission in blockchain social network," Information Science, vol. 39, pp. 35-40, 2021.

[10] B. Jie and D. Yanhui, "Data mining framework for sensitive information of large-scale social online games based on blockchain," Journal of Xi'an University of Technology, vol. 2, pp. 1-6, 2021.

[11] W. Xiwei, Z. Liu, H. Bo, and W. Yanan, "Research on network rumor screening model and simulation based on blockchain," Journal of Infection, vol. 40, pp. 194-203, 2021.

[12] G. Sulin, H. Wei, and L. Ji, "Research on the impact of blockchain technology on the willingness of public opinion users to accept information," IntelliJ IDEA, vol. 39, pp. 130136, 2020.

[13] Z. Weichong, W. Fang, and Z. Hong, "Multi source information fusion for emerging technology development trend identification-taking blockchain as an example," Journal of Information Technology, vol. 38, pp. 1166-1176, 2019.

[14] Z. Dan, W. Xiwei, H. Jieping, and Y. Wencong, "Research on the characteristics and laws of network public opinion information dissemination in the blockchain environment," IntelliJ IDEA, vol. 37, pp. 127-133, 2018.

[15] A. A. Khan, M Uddin, A. Shaikh, A. A. Laghari, and A. Rajput, "MF-ledger: blockchain hyperledger sawtooth-enabled novel and secure multimedia chain of custody forensic investigation architecture," Journal of IEEE Access, vol. 99, p. 1, 2021.

[16] Y. Yuanyuan, W. Xi, and Y. Ying, "Exploration of multisource network public opinion data based on blockchain incentive mechanism," Journal of Culture and Communication, vol. 9, no. 02, pp. 63-67, 2020.

[17] X. Jiabiao and C. Qian, "Blockchain technology and network rumor governance in the era of we media," Contemporary Communications, vol. 02, pp. 97-99, 2021. 